\title{
A violência urbana contra crianças e adolescentes em Belo Horizonte: uma história contada através dos traumas maxilofaciais
}

\author{
I ${ }^{1}$ Carlos José de Paula Silva, ${ }^{2}$ Efigênia Ferreira e Ferreira, ${ }^{3}$ Liliam Pacheco \\ Pinto de Paula, ${ }^{4}$ Marcelo Drummond Naves, ${ }^{5}$ Andreia Maria Duarte Vargas, \\ ${ }^{6}$ Patricia Maria Pereira Araujo Zarzar I
}

Resumo: Os traumas maxilofaciais decorrentes da violência contra crianças e adolescentes impactam suas vidas, física e psiquicamente, pelas deformidades que podem provocar e pela exposição da lesão na face das vítimas. O objetivo deste trabalho é identificar a prevalência dos traumas maxilofaciais em crianças e adolescentes decorrentes da violência urbana em Belo Horizonte- Brasil. O estudo foi conduzido no Hospital Municipal Odilon Behrens, único hospital municipal de referência nesse tipo de atendimento em Belo Horizonte. Coletaram-se os registros de vítimas atendidas de janeiro a dezembro de 2007. O principal evento de violência sofrido entre crianças e adolescentes foi agressão física, 44,2\% e 64,7\%, respectivamente. Entre as crianças, o tipo de trauma mais comum foi o trauma dentoalveolar $(53,8 \%)$, e entre os adolescentes, trauma de tecidos moles (47,5\%). O maior número de ocorrências se deu no período noturno: crianças $(84,6 \%)$ e adolescentes $(74,8 \%)$. O gênero mais vitimado foi o masculino, crianças $(63,5 \%)$ e adolescentes $(68,3 \%)$. Estratégias apropriadas para identificação do evento de violência e do agressor são necessários para que melhor sejam planejados mecanismos de proteção da criança e do adolescente, uma vez que a violência sofrida por crianças e adolescentes no Brasil, considerando a complexidade dessa fase da vida, assume um quadro sombrio, desconstruindo o desenvolvimento, a sociabilidade e comprometendo a visão das vítimas sobre si mesmas e sobre o mundo que as cercam.

> Palavras-chave: trauma maxilofacial; violência; crianças; adolescentes.
${ }^{1}$ Doutorando em Saúde Coletiva da Escola de Odontologia da Universidade Federal de Minas Gerais (UFMG). Mestre em Saúde Coletiva pela UFMG. Especialista em Saúde Coletiva pela Universidade Federal de Juiz de Fora (UFJF). Endereço eletrônico: case.odo@gmail.com

2 Doutora em Epidemiologia. Professora do Departamento de Odontologia Social e Preventiva, Faculdade de Odontologia da Universidade Federal de Minas Gerais - UFMG. Endereço eletrônico: efigenia@gmail.com

${ }^{3}$ Psicóloga, mestre em Ciências da Informação pela UFMG. Endereço eletrônico: liampacheco@hotmail.com

${ }^{4}$ Doutor em Estomatologia. Professor do Departamento de Clinica, Patologia e Cirurgia odontológica, Faculdade de Odontologia da UFMG. Endereço eletrônico: madruna@uol.com.br

${ }^{5}$ Professora da UFMG, Faculdade de Odontologia, Departamento de Odontologia Social e Preventiva. Endereço eletrônico: vargasnt@task.com.br

${ }^{6}$ Professora da UFMG, Faculdade de Odontologia, Departamento de Odontologia Social e Preventiva. Endereço eletrônico: patyzarzar@hotmail.com

Recebido em: 03/08/2010. Aprovado em: 01/06/2011. 
A violência é analisada antropologicamente como um filtro que possibilita esclarecer certos aspectos do mundo social, por denotar as características do grupo social e revelar seus significados no contexto das relações sociais. É considerada, portanto, um fenômeno social (GULLO, 1998).

A violência é um fenômeno que permite variadas conceituações. Sendo a violência urbana uma forma particular desse fenômeno, ela é o resultado da relação dos habitantes com o espaço urbano e de suas relações sociais (RIBEIRO; CHAVEIRO, 2007). Essa violência se deriva da organização desse espaço e surge como resultado dos conflitos e problemas urbanos (PINHEIRO; ALMEIDA, 2003). Ela modifica o cotidiano dos habitantes das cidades; subverte e desvirtua a função destas; drena seus recursos e ceifa vidas (PAVIANI; FERREIRA; BARRETO, 2005). Esses autores a consideram um fenômeno social decorrente das características subjetivas individuais da vítima e do agressor, bem como dos processos macrossociais que se articulam e se interagem dinamicamente. Trata-se de uma "interação" entre indivíduos situados em uma dada estrutura social, ocupando papéis sociais e orientados por valores que definem e modelam as possibilidades dessa interação. A violência urbana tornou-se constante no cotidiano dos brasileiros, produzindo um grande número de vítimas e sequelas físicas e emocionais, constituindo-se em um problema de saúde pública.

Os traumas maxilofaciais decorrentes da violência contra crianças e adolescentes impactam suas vidas, física e psiquicamente, pelas deformidades que podem provocar e pela exposição da lesão na face das vítimas. Especificamente, os casos de trauma maxilofacial em crianças e adolescentes têm exigido maior atenção, principalmente em função do aumento dos episódios de violência doméstica, que, na grande maioria, não chegam ao conhecimento das autoridades policiais e de saúde (RIBEIRO \& GOES, 2006). Essa violência pode ser considerada como toda ação ou omissão realizada por indivíduos, grupos, classes ou nações que ocasionem danos físicos, emocionais, morais e espirituais a si próprios ou aos outros (BRASIL, 2001).

Para crianças e adolescentes a violência pode ser representada por toda ação ou omissão capaz de provocar lesões, danos ou transtornos ao desenvolvimento integral, envolvendo eventos com uma relação assimétrica e desigual de poder manifestada pela força física, poder econômico ou político (ASSIS; DESLANDES; 
SANTOS, 2005). A vitimização de crianças e adolescentes por acidentes e violências é classificada como "causas externas" segundo a CID 10 - Classificação Estatística Internacional de Doenças e Problemas Relacionados à Saúde (OMS, 1995). Em Belo Horizonte, especificamente, a taxa de mortalidade por causas externas em 2000, para crianças de 1 a 9 anos, chegou a 12,9 por 100.000 habitantes. Para os adolescentes de 10 a 14 anos, 20,0 por 100.000; e na faixa etária de 15 a 19 anos chegou à taxa de 82,0 por 100.000 habitantes (BRASIL, 2005).

Esses números demonstram a gravidade do quadro da violência contra crianças e adolescentes nas grandes cidades brasileiras. Entretanto, os números apontados refletem apenas os casos com desfecho fatal. A infância é uma fase $\mathrm{da}$ vida extremamente delicada e requer grandes investimentos afetivos e de suporte social. Os cuidados prestados à criança influenciarão sua possibilidade de sobrevivência e de qualidade de vida, e servirão como um espelho de valores no qual ela irá se mirar para formar suas ideias sobre si e sobre o mundo (DESLANDES; ASSIS; SANTOS, 2005; WINNICOTT, 1987). A adolescência é vista em diversas culturas e épocas como importante momento de domínio das regras e dos valores da vida social, de ganho da autonomia, de maturação física e psíquica e de gradativa incorporação de papéis sociais do mundo adulto e, enquanto continuidade da infância, normalmente seu desfecho e vivência surge como consequência de experiências vividas nessa fase que a antecede.

Crianças e adolescentes têm acumulado tipos diferentes de violência em suas histórias de vida, o que potencializa ainda mais a vivência negativa dessa violência e faz com que estes passem a desejar estar do outro lado do contexto: de vítimas a agressores. Dessa forma, a violência contra crianças e adolescentes em suas várias formas de manifestação e complexidade reafirma a importância da abordagem do tema para a saúde coletiva, bem como de não negligenciá-lo, sob pena de estarmos comprometendo a formação das gerações futuras e a construção de um país mais justo e seguro.

Este enfoque foi dado considerando a existência de poucos estudos que abordem os sinais de violência na face de crianças e adolescentes e pela repercussão dessa violência seja pela implicação social dos traumas considerando idade das vítimas e seja pelo significado simbólico da face. Assim, o presente estudo objetivou, através dos casos de trauma maxilofacial, analisar os eventos de violência contra crianças e adolescentes. 
O presente estudo foi desenvolvido na cidade de Belo Horizonte-MG. Em 2007, ano de referência da pesquisa, a cidade apresentava uma população total de 2.412.937 de habitantes, com economia baseada no comércio e serviços, e um IDH igual a 0,839. Foi escolhido para esta pesquisa o Hospital Municipal Odilon Behrens (HMOB), por ser um hospital especializado nesse tipo de atendimento.

Foi efetuada uma análise retrospectiva de todos os atendimentos realizados no Serviço de Cirurgia e Traumatologia Bucomaxilofacial do HMOB. As informações foram coletadas nos livros de registro dos plantôes diurno e noturno. Foram incluídos no estudo todos os casos registrados no período de janeiro a dezembro de 2007. Foram classificadas como crianças, os indivíduos até 11 anos de idade e como adolescentes os indivíduos com idade entre 12 a 18 anos (BRASIL, 1990). Os eventos de violência urbana foram classificados em: acidentes automobilísticos (AAT), acidentes motociclísticos (AMT), agressão física (AGF), agressão por arma de fogo (AAF) e atropelamento (ATP).

Consideraram-se como variável dependente os eventos de violência, e como variáveis independentes, o tipo de trauma, data, período do dia, idade e gênero da vítima. Foram consideradas como fraturas simples (FS) aquelas que apresentaram apenas um traço de fratura, e fraturas múltiplas (FM) as que apresentaram dois ou mais traços (MONTOVANI; CAMPOS; GOMES, et al., 2006).

Os traumas de tecido mole (TTM) foram os que apresentaram como características as lacerações, cortes, abrasōes com perda de substância (MANGANELLO- SOUZA, 2006). Os traumas dentoalveolares (TDA) foram os ocorridos nos dentes e tecidos de sustentação (ANDREASEN, 1984). Os traumas cranioencefálicos (TCE) são caracterizados pelo comprometimento neurológico (RIBAS, 2006).

As análises envolveram estatísticas descritivas, análise bivariada, teste quiquadrado de Pearson, exato de Fisher, com nível de significância $\mathrm{p}<0,05$. Para identificação das variáveis associadas, utilizou-se o valor residual ajustado $(\mathrm{z}>1,96)$. O software utilizado foi o SPSS versão 17.0. A pesquisa foi aprovada pelos Comitês de Ética em Pesquisa da Universidade Federal de Minas Gerais e do Hospital Municipal Odilon Behrens, com o registro no ETIC 352/08. 
No período compreendido entre janeiro a dezembro de 2007, foram atendidos no Serviço de Cirurgia e Traumatologia Bucomaxilofacial, do Hospital Municipal Odilon Behrens, 191 casos que se referiam à violência contra crianças e adolescentes. Os adolescentes foram vítimas em 139 eventos de violência (72,8\%), e as crianças, em 52 casos (27,2\%). Em relação ao gênero, foi observado que, entre as crianças, prevaleceu o masculino, com 33 casos, $(63,5 \%)$ sobre o feminino com 19 casos (36,5\%). Entre os adolescentes, 95 casos $(68,3 \%)$ ocorreram no gênero masculino e 44 casos $(31,7 \%)$ no feminino.

$\mathrm{Na}$ distribuição dos eventos de violência observou-se a predominância de AGF, com 23 casos $(44,2 \%)$ e 90 casos $(64,7 \%)$, em crianças e adolescentes respectivamente. Entre as crianças, tem-se ainda o ATP como segunda maior causa de trauma, com 18 casos (34,6\%). Já nos adolescentes, os AAT, com 30 casos $(21,6 \%)$. Ocorreu também um caso de AAF (0,7\%) entre os adolescentes.

Considerando os tipos de trauma, nas crianças, o principal tipo foi o TDA com 28 casos $(53,8 \%)$, seguido por TTM com 20 casos $(38,5 \%)$. Entre os adolescentes, o TTM prevaleceu com 66 casos (47,5\%), seguido por FS, com 35 casos $(25,2 \%)$. Entre os adolescentes ocorreram também dois casos de TCE $(1,4 \%)$ (Ttabela 1$)$.

Com relação à distribuição dos traumas maxilofaciais sofridos por crianças e adolescentes segundo os períodos do dia, os resultados mostram uma predominância dos traumas no período noturno. Nesse período, ocorreram 44 casos $(84,6 \%)$ em crianças e 104 casos em adolescentes. No período diurno as ocorrências foram de oito casos $(15,4 \%)$ em crianças e 35 casos $(25,2 \%)$ em adolescentes.

A análise bivariada revelou existir diferença estatisticamente significativa entre crianças e adolescentes e os eventos de violência. As crianças estavam associadas aos atropelamentos $(\mathrm{p}<0,001)$ e os adolescentes à agressão física $(\mathrm{p}<0,001)$. Foi verificada também diferença estatisticamente significativa entre os grupos e os tipos de trauma, nos quais as crianças estavam associadas aos traumas dentoalveolares $(\mathrm{p}<0,001)$ e os adolescentes às fraturas simples $(\mathrm{p}<0,001)$. 
TABELA 1: Relação entre crianças e adolescentes quanto aos eventos de violência, tipo de trauma, gênero e período do dia

\begin{tabular}{l|c|c|c|c|c}
\hline \multirow{2}{*}{} & \multicolumn{2}{|c|}{ Crianças } & \multicolumn{2}{c|}{ Adolescentes } & \multirow{2}{*}{ Valor p } \\
\cline { 2 - 5 } & $\mathbf{n}(\%)$ & R.aj. & $\mathbf{n}(\%)$ & R.aj. & \\
\cline { 1 - 4 } Etiologia & $9(17,3)$ & $-0,7$ & $30(21,6)$ & 0,7 & \\
\hline Ac.automobilístico & $2(3,8)$ & $-1,4$ & $14(10,1)$ & 1,4 & \\
\cline { 1 - 4 } Ac. motociclístico & $23(44,2)$ & $-2,6$ & $90(64,7)$ & $2,6^{*}$ & \multirow{2}{*}{$<0,001^{* *}$} \\
\hline Agressão física & $18(34,6)$ & $6,1^{*}$ & $4(2,9)$ & $-6,1$ & \\
\hline Atropelamento & $0(00,0)$ & $-0,6$ & $1(0,7)$ & 0,6 & \\
\hline Arma de fogo & $52(100,0)$ & & $139(100,0)$ & & \\
\hline Total & & & & \\
\hline
\end{tabular}

\begin{tabular}{|c|c|c|c|c|c|}
\hline \multicolumn{5}{|l|}{ Trauma } & \multirow{7}{*}{$<0,001^{* *}$} \\
\hline Tecidos moles & $20(38,5)$ & $-1,1$ & $66(47,5)$ & 1,1 & \\
\hline Fratura múltipla & $0(00,0)$ & $-1,4$ & $5(3,6)$ & 1,4 & \\
\hline Fratura simples & $4(7,7)$ & $-2,7$ & $35(25,2)$ & $2,7^{*}$ & \\
\hline Dentoalveolar & $28(53,8)$ & $4,2^{*}$ & $31(22,3)$ & $-4,2$ & \\
\hline Cranioencefálico & $0(00,0)$ & $-0,9$ & $2(1,4)$ & 0,9 & \\
\hline Total & $52(100,0)$ & & $139(100,0)$ & & \\
\hline
\end{tabular}

\begin{tabular}{l|c|c|c|c|c}
\hline \multicolumn{1}{l|}{ Gênero } & $33(63,5)$ & $-0,6$ & $95(68,3)$ & 0,6 & \multirow{2}{*}{$0,604^{* * *}$} \\
\cline { 1 - 4 } Masculino & $19(36,5)$ & 0,6 & $44(31,7)$ & $-0,6$ & \\
\hline Feminino & $52(100,0)$ & & $139(100,0)$ & & \\
\hline Total & & & \\
\hline
\end{tabular}

\begin{tabular}{l|c|c|c|c|c}
\hline \multicolumn{1}{l|}{ Período } & $8(15,4)$ & $-1,4$ & $35(25,2)$ & 1,4 & \multirow{2}{*}{$0,176^{* * *}$} \\
\cline { 1 - 4 } Diurno & $44(84,6)$ & 1,4 & $104(74,8)$ & $-1,4$ & \\
\cline { 1 - 4 } Noturno & $52(100,0)$ & & $139(100,0)$ & & \\
\hline Total & & & \\
\hline
\end{tabular}

* valor residual ajustado $>1,96$.

** valor p referente ao teste exato de Fisher.

*** valor $\mathrm{p}$ referente ao teste qui-quadrado de Pearson. 
As características epidemiológicas dos eventos de violência podem variar dependendo dos aspectos demográficos, localização geográfica, aspectos culturais e condições socioeconômicas da população estudada (BAMJEE et al., 1996). Os dados exibidos mostram uma predominância dos adolescentes sobre as crianças: 139 e 52 casos respectivamente. Em outros estudos, independentemente dos fatores etiológicos encontrados, essa tendência também foi percebida (CHRCANOVIC et al., 2004; MONTOVANI et al., 2006; SARMENTO; CAMPOS; SANTOS, 2007; MENEZES et al., 2007). A exposição aos traumas maxilofaciais apresenta como característica aumento gradual até 18 anos de idade, podendo apresentar pequenas variaçóes de estudo para estudo. Nesses estudos verifica-se uma informação importante, pois se percebe que, à medida que houve passagem da infância para a adolescência, a frequência dos eventos de violência e dos traumas aumentou. Dessa forma, o envolvimento dos adultos com a violência, seja como vítima ou agressor, fato apontado e discutido amplamente na literatura, pode significar um resultado trágico de todas as experiências vivenciadas na infância e adolescência. Segundo Paula et al. (2008), o fato de crianças e adolescentes presenciarem algum tipo de violência é fator de risco para doença mental. Para as crianças, especificamente, considerando a violência doméstica, esse risco é três vezes maior quando comparada à violência urbana.

Ademais a exposição frequente à violência na infância pode levar a uma adaptação negativa, causando internalização de problemas como ansiedade, depressão e baixa auto-estima (SALZINGER et al., 2008). Nesse sentido, a violência pode ter efeitos diferentes em idades diferentes, e pode também comprometer a habilidade das vítimas no enfrentamento de desafios durante a vida, afetando o desenvolvimento da criança do ponto de vista emocional (MARGOLIN; GORDIS, 2000). Conclusão semelhante foi apontada por Browne e Winkelman (2007) e Lange et al. (2004): os autores citaram que os traumas na infância estavam intimamente relacionados ao desajuste psicológico tardio e aos conflitos em relacionamentos na idade adulta.

No tocante ao gênero, percebem-se nitidamente diferenças no que diz respeito à vitimização de crianças e adolescentes. As meninas, talvez por serem mais protegidas, e por apresentarem comportamento menos agressivo, foram menos 
vitimadas. Os meninos, nos dois grupos, foram maioria entre as vítimas, sugerindo que estes estão mais expostos aos fatores de risco para os traumas maxilofaciais. Essa condição é revivida na maioridade, em que o gênero masculino predomina como vítima e agressor (MONTOVANI et al., 2006; BAMJEE et al., 1996; CHRCANOVIC et al., 2004; SARMENTO; CAMPOS; SANTOS, 2007; MENEZES, et al., 2007; MACEDO et al., 2008; GONDOLA et al., 2006; PORTOLAN; TORRIANI, 2005; FALCÃO; SEGUNDO; SILVEIRA, 2005).

O estudo revela o predomínio da agressão física tanto entre as crianças quanto entre os adolescentes, com 23 casos (44,2\%) e 90 casos (64,7\%) respectivamente. A violência contra crianças e adolescentes é culturalmente aceita em muitos países, e essa agressão é vista com o propósito disciplinar (LIZUKA et al., 1995). Em relação às crianças, foi visto um alto índice de vitimização por ATP, com $(34,6 \%)$, sendo a segunda causa de traumas para esse grupo. Isso pode remeter ao descuido com a criança, seja quando acompanhada por um adulto, ou por deixá-la sob seus próprios cuidados. Entre os adolescentes, observou-se como segunda causa de trauma os acidentes com AAT (21,6\%), o que pode sugerir que o adolescente, nesses casos, se encontrava acompanhado por adultos que o expuseram a esse tipo de violência, visto que ainda não apresentava idade que possibilitasse ser ele o condutor do veículo ou, no extremo, o adolescente conduzia um veículo sem habilitação.

Informação importante foi a ocorrência do evento de violência decorrente de AAF (um caso) entre os adolescentes, considerando o poder destrutivo dessas armas. Esse dado entre adolescentes pode sugerir tanto a exposição da vítima a esse tipo de violência como a sua participação no ato da violência. Nesse sentido, a arma de fogo afirma o caráter inequívoco da intencionalidade de matar. Erickson et al. (2006) concluíram, em estudo desenvolvido em quatro grandes centros urbanos, Toronto, Amsterdam, Montreal e Filadélfia, que o risco de perpetração ou vitimização por violência era alto nessas cidades justamente pela utilização de arma de fogo, principalmente relacionadas com a ação de gangues de adolescentes. Os autores citam ainda que esse tipo de violência tem-se estendido além das fronteiras e culturas. $\mathrm{O}$ trauma maxilofacial provocado por arma de fogo obedece a uma escala crescente de severidade quando se observa a idade da vítima: as crianças são geralmente acometidas de trauma de tecidos moles, enquanto os adolescentes enfrentam desordens mais severas do complexo facial (GASSNER et al., 2003). 
A questão da violência infantil tem sido abordada e tratada por leis desde 1948, com a instituição da Declaração Universal dos Direitos Humanos (ONU, 1948), artigos 3 e 5; de 1959, com a elaboração da Declaração Universal dos Direitos da Criança (DUDC, 1959) princípio 9; de 1990, pela Convenção sobre os Direitos da Criança (BRASIL, 1990) (arts. 19.1 e 2, 9, 34, 35, 36, e, 39); e ainda desde a Constituição Federal de 1988 (BRASIL, 1988) (art. 226). Em 1990, foi criado o Estatuto da Criança e do Adolescente (ECA) que passa a vê-los como sujeitos de direitos, pessoas em condiçôes peculiares de desenvolvimento, que requerem proteção e prioridade absoluta no nível das políticas públicas. O ECA oferece estratégias para garantir os direitos da criança e do adolescente, e propõe medidas de intervenção em relação à família agressora, protegendo a vítima e estabelecendo a necessidade de prevenção do fenômeno.

Após a criação do ECA, a notificação da violência, ou sua suspeita, passou a ser obrigatória. No entanto, essa ainda não é a realidade da sociedade brasileira, seja por medo de envolvimento, seja por proteção do membro agressor, ou por medo de represálias. Outros fatores encobrem a realidade da violência contra crianças e adolescentes: ausência de percepção de algumas formas de violência, culturalmente aceitas, ausência da denúncia da violência doméstica pela família (disfarce dos casos, nos quais a lesão da criança ou adolescente é, via de regra, referida a tombos, brigas, acidentes, entre outros). Esses fatores possibilitam o pacto do silêncio, a tolerância social e a impunidade.

Rodrigues (1999), em estudo sobre crianças e adolescentes atendidos no Hospital Universitário Regional do Norte do Paraná, ressalta que as equipes assistenciais não se ocupam das causas da violência contra a criança, e que ocorrem somente conversas isoladas buscando entender o que realmente se passou com ela. $\mathrm{O}$ autor ressalta que não foram tomadas medidas formais com relação à violência sofrida pela criança: apenas a assistência social tenta protegê-la afastando-a do agressor ou indicando um adulto protetor dentro da residência onde reside. Ressalta ainda que houve deficiência no registro específico da identificação das situações de violência, e a dificuldade de acesso aos prontuários. O desconhecimento do ECA foi um fator que contribuiu para o não envolvimento dos profissionais. $\mathrm{O}$ autor conclui que, na realidade do hospital estudado, a desarticulação entre os profissionais envolvidos no atendimento às vítimas de violência culminou na dificuldade de identificação 
1112 das situaçôes de violência - pela ausência de análise sob múltiplo enfoque - e na limitação do atendimento, pela inexistência de um trabalho interdisciplinar.

Fato também verificado no presente estudo, pois os livros de registro consultados apresentavam informações apenas relativas ao período do dia, à idade da vítima, ao seu gênero, ao tipo de trauma, à etiologia, aos sinais e sintomas, ao procedimento realizado e à data do atendimento. No Brasil, parece não haver a compreensão por parte dos profissionais sobre a necessidade de construção e tratamento de informações com objetivos epidemiológicos. Ocorrem apenas os registros quantitativos da rotina de atendimentos. Não foram identificados registros sobre o agressor, sobre a relação do agressor com a vítima, sobre local onde a violência ocorreu, etc. Dados estes que poderiam ser úteis na associação entre os traumas e a violência contra crianças e adolescentes. No âmbito desse trabalho, quanto ao conhecimento ou não do ECA, nada se pode afirmar, pois isso não foi questionado aos profissionais. Mas uma inferência pode ser feita: o hospital encontra-se próximo a uma favela ou "aglomerado", como são conhecidas em Belo Horizonte, fato que pode contribuir para o não envolvimento dos profissionais, que poderiam temer pela própria segurança. O que tem ficado cada vez mais claro é que muitas vezes no Brasil a violência contra crianças e adolescentes é tratada como algo privado, recebendo relativa atenção apenas nos casos em que a vítima consegue clamar por auxílio (DESLANDES, S.F.; ASSIS, S.G.; SANTOS, N.C, 2005). Uma situação interessante ocorreu nas conversas informais estabelecidas com os profissionais durante a coleta dos dados. Eles relataram que, muitas vezes, percebiam que os acompanhantes (sejam familiares ou não) foram os agressores das vítimas, mas não assumiam esse lugar para o profissional.

No estudo, observando os resultados da distribuição das vítimas quanto aos tipos de trauma encontrados, concluiu-se que, entre as crianças, os TDA, foram maioria com 28 casos (53,8\%), seguidos pelos casos de TTM: 20 casos (38,5\%). Os adolescentes sofreram em maioria TTM, com 66 casos (47,5\%), seguidos pelos FS: 35 casos (25,26\%). Os traumas de tecidos moles são reconhecidos como mais frequentes em estudos internacionais (BAMJEE, et al., 1996; RAHMAN et al., 2007; PRIGOZEN et al., 2006). Independentemente da gravidade ou não dos eventos de violência sofridos, ressalta-se o impacto na experiência de vida e no desenvolvimento emocional das vítimas, e pior, muitas situaçôes dificilmente se 
transformam em números, como nos casos de abuso sexual, que podem permanecer ocultos dentro de uma pseudoproteção ou omissão do núcleo familiar.

Considerando os tipos de traumas sofridos por crianças e adolescentes no estudo, pode-se levantar a hipótese de que os adolescentes podem ser, nesses casos, tanto as vítimas como os próprios agressores. Não há a possibilidade de afirmação pelo fato de os prontuários não contemplarem informações sobre o agressor e o motivo da violência, o que pode ser apontado como um possível viés do presente estudo. Outra hipótese é de que a violência pode estar ocorrendo em casa, no interior das residências. Um aspecto muito relatado pelos profissionais do hospital onde foram coletadas as informações desse estudo foi o de que muitas vítimas buscaram atendimento, acompanhados por seus responsáveis. Estes relatavam que a criança ou o adolescente teria sofrido queda da própria altura. Contudo, ao se avaliar a extensão das lesões percebia-se que eram desproporcionais, como no caso das fraturas. Entretanto, não existem mecanismos no serviço para identificar a veracidade do que é informado pelos responsáveis. Por isso, os profissionais devem estar atentos para as características das lesões quando as etiologias não são devidamente identificadas, deve-se observar a possibilidade da existência de lesões em diferentes estágios de cura, o que pode significar exposição frequente a eventos de violência (FENTON; BOUQUOT; UNKEL, 2000).

Quando comparados crianças e adolescentes, percebe-se que os adolescentes apresentaram um padrão mais importante quanto à abrangência de tipos de trauma e de eventos de violência, podendo concluir que esse grupo está mais exposto ao risco e a um aumento na prevalência dos traumas maxilofaciais, o que pode ser demonstrado pelos casos de TCE e AAF encontrados exclusivamente entre os adolescentes. Quanto mais o adolescente for exposto a trajetórias de violência e agressão física, maior a possibilidade de se envolver em atos delinquentes mais sérios e comportamento agressivo (NAGIN; TREMBLAY, 2003; VILHENA; MAIA, 2002), o que pode ser um alerta para o desenvolvimento de políticas. Estas devem assegurar o desenvolvimento do adolescente em um ambiente que lhe proporcione segurança e condições de investimento em aspectos positivos da vida, que lhe renderão possibilidades de construir uma trajetória mais digna para si e menos danosa para a sociedade.

Os TDA e TTM podem resultar em complicações estéticas e funcionais afetando física e emocionalmente as vítimas, e isso pode ser potencializado 
quando o trauma ocorre nos dentes anteriores (ARAÚJO; VALERA, 1999). As crianças apresentam uma cicatrização mais rápida dos tecidos, enquanto nos adolescentes o surgimento de cicatrizações hipertróficas é frequente (MODOLIN et al., 2006). Esse tipo de cicatrização tecidual resulta da proliferação exagerada dos tecidos, podendo levar a um comprometimento estético importante influenciando diretamente na auto-estima das vítimas. Não foi encontrada no estudo uma grande frequência de fraturas na face de crianças. Conclusão semelhante foi apontada em outros estudos (ADEKEYE, 1980; ZACHARIADES; PAPAVASSILIOU; KOUMOURA, 1990).

A análise dos dados quanto ao período do dia, mostra que os dois grupos foram vítimas predominantemente no período noturno. Pode-se supor que as crianças e os adolescentes do estudo tenham sido agredidos em seus próprios lares ou foram envolvidos em situações que os expuseram à violência nesse período do dia. Pode-se considerar que crianças, geralmente, se encontram em casa nesse período do dia. O fato de terem ocorrido 44 casos $(84,6 \%)$ de violência a crianças no período noturno nos leva a pensar na possibilidade destas terem sofrido o trauma posteriormente à chegada dos pais em casa, o que se configuraria como violência doméstica, lembrando que, quanto ao evento de violência prevaleceu a agressão física (44,2\%). Ademais, o período noturno coincide muitas vezes com o horário em que muitos homens chegam alcoolizados a suas residências e usam de violência contra filhos, esposas ou companheiras (STRON, 1992).

No entanto, não se pode afirmar que a realidade exposta pelos dados se refere à displicência da família ou à sua agressão contra a criança e o adolescente, pois o prontuário do hospital não contém informações específicas e essenciais para essa conclusão, levando à possibilidade de subnotificações. A ocorrência dessas subnotificações, somada à ausência de dados, comuns em estudos sobre violência, pode ser apontada como limitação do estudo. Outra limitação é o possível viés de informaçãa, pois pode haver uma sub-revelação do ocorrido. Isso pode esconder o fato de que os traumas maxilofaciais em crianças e adolescentes, muitas vezes, são fruto de violência doméstica. A situação de vulnerabilidade, muitas vezes vivida pelos pais, leva as crianças e jovens a serem vítimas e agressores em potencial, vindo futuramente a ocuparem os lugares dos pais na rede de geração e expansão da violência na sociedade. Essa ideia é compartilhada por outros autores como Adorno, Lima e Bordini (1999), Cyrulnik (2007) e Ridley (2000). Winnicott 
(1987, p.257) afirma que tudo que leva as pessoas aos tribunais (ou hospícios, tanto importa para o caso) tem o seu equivalente normal na infância, na relação entre a criança e seu próprio lar. Segundo o autor, caso ocorra a preocupação materna primária e o pai assuma seu lugar em dar suporte emocional para a mãe, será criada uma atmosfera de cuidado e proteção à criança que contribuirá para seu desenvolvimento sadio. Caso contrário, a criança desenvolverá um falso selfe poderá repetir os comportamentos disfuncionais dos pais.

Percebem-se necessários, a partir dos fatos apresentados, uma mudança na estruturação dos prontuários e um trabalho de conscientização dos profissionais para que estudos posteriores a este possam traçar um quadro mais fiel da realidade da violência sofrida por crianças e adolescentes nas cidades brasileiras. É, portanto, relevante trazer para o espaço de discussão, nas ciências da saúde, as condições motivadoras dessa violência.

\section{Conclusões}

Em conclusão, o predomínio dos casos de agressão física como evento de violência contra crianças e adolescentes encontrados neste estudo são coincidentes com os de outros estudos. O perfil da vítima para agressão física também é compatível, atingindo, em sua maioria, jovens do sexo masculino.Os casos de atropelamento entre as crianças, apontados no estudo como significativos, bem como a forma mais prevalente do tipo de trauma, são dados que merecem atenção quanto ao cuidado dispensado a estas pelos seus cuidadores. Estratégias apropriadas para identificação dos eventos de violência e a identificação do agressor são necessárias para que melhor sejam planejados mecanismos de proteção a crianças e adolescentes.

\section{Referências}

ADEKEYE, E.O. Pediatric fractures of the facial skeleton: a survey of 85 cases from Kaduma, Nigeria. Journal of Oral Surgery, v. 38, p.355, 1980.

ADORNO, S.; LIMA, R.S.; BORDINI, E.B.T. $O$ adolecente na criminalidade urbana em São Paulo-Brasilia: Ministerio da Justiça, Secretaria de Estado de Direitos Humanos, 1999. ANDREASEN, J.O. Lesiones Traumáticas de los Dientes. 3. ed. Trad. G. M. Herrero. Barcelona: Labor, 1984. p.225-283.

ARAÚJO, M.A.M.; VALERA, M.C. Tratamento clínico dos traumatismos dentários. Série EAP/ APCD.São Paulo: Artes Médicas; 1999. 
ASSIS, S.G.; DESLANDES, S.F.; SANTOS, N.C. Violência na adolescência: sementes e frutos de uma sociedade desigual. In Impacto da Violência na Saúde dos Brasileiros, Ministério da Saúde, Secretaria de Vigilância em Saúde, Departamento de Análise de Situação de Saúde, Brasil, 2005.

BAMJEE, Y. et al. Maxillofacial injuries in a group of South Africans under 18 years of age. Britsh Journal of Oral and Maxillofacial Surgery, Edinburgh, v.34, n. 4, p.298-302, Aug. 1996. BRASIL. Ministério da Justiça. Plano Nacional de Enfrentamento da Violência Sexual Infanto-Juvenil. Brasília: Ministério da Justiça, Secretaria de Estado de Direitos Humanos e Departamento da Criança e do Adolescente, 2001.

BRASIL. Ministério da Saúde. Secretaria de Vigilância em Saúde. Departamento de Análise de Situação de Saúde. Impacto da Violência na Saúde dos Brasileiros. Brasil, 2005.

BRASIL. ECA Estatuto da Criança e do Adolescente. Lei no 8.069 de 13/07/90. Brasília: Conselho dos Direitos da Criança e do Adolescente, 1995.

BRASIL. Convenção sobre direitos da criança. Brasília: Conselho dos Direitos da Criança e do Adolescente, 1990.

BRASIL. Congresso Nacional. Constituição da República Federativa do Brasil: promulgada em 05 de outubro de 1988. Brasília: Senado Federal, 1988.

BROWNE, C.; WINKELMAN, C. The Effect of Childhood Trauma on Later psycological Adjustament. Journal of Interpersonal Violence, v. 22, n. 6, p. 684-697, 2007.

CHRCANOVIC, B.R. et al. Facial fractures: a 1-year retrospective study in a hospital in Belo Horizonte. Brazilian oral research, v. 18, n.4, p. 322-328.2004.

CYRULNIK, B. Os alimentos afetivos: o amor que nos cura. São Paulo: Martins Fontes, 2007. Declaração Universal dos Direitos Humanos, Organização das Naçôes Unidas, resolução 217 A (III). 10 de Dezembro de 1948. Disponível em: http://www.mj.gov.br/sedh/ct/legis_ intern/ddh_bib_inter_universal.htm Acesso em: 23 dez 2008.

Declaração Universal dos Direitos da Criança, 1959. 16. Declaração Universal dos Direitos Humanos, 1948. Disponível em: http:/www.pge.sp.gov.br/centrodeestudos/ bibliotecavirtual/instrumentos/2decla.htm Acesso em: 21 set 2008.

DESLANDES, S.F.; ASSIS, S.G.; SANTOS, N.C. Violência envolvendo crianças no Brasil: um plural estruturado e estruturante. In: Impacto da Violência na Saúde dos Brasileiros. Ministério da Saúde, Secretaria de Vigilância em Saúde, Departamento de Análise de Situação de Saúde, Brasil, 2005.

ERICKSON, P.G. et al. Girls and weapons: An International Study of the perpetration of Violence. Journal of Urban Health, v. 83, n. 5, p.788-801, Aug. 2006. 
FALCÃO, M.F.L.; SEGUNDO, A.V.L.; SILVEIRA, M.M.F. Estudo epidemiológico de 1758 fraturas faciais tratadas no hospital da restauração, recife-PE. Revista de Cirurgia Traumatologia Bucomaxilofacial. Camaragibe. V. 5, n.3, p 65-72, jul/ set.2005.

FENTON, S.J.; BOUQUOT, J.E.; UNKEL, J.H. Orofacial considerations for pediatric, adult, and elderly victims of abuse. Emerg. Med Clin. North Americam, Philadelphia, v. 18, n. 3, p.601-6017, August 2000.

GASSNER, R.; TULI, T.; HACHL, O. et al. Crânio-maxillofacial trauma: a 10 year review of 9543 cases with 21067 injuries. Journal of Craniomaxillofacial Surgery, v. 31, p. 51-61, 2003. GONDOLA, A.O. et al. Epidemiologia das fraturas zigomáticas: uma análise de 10 anos. Revista Odonto Ciência. Porto Alegre, v. 21, n. 52, abril/ junho, 2006.

GULLO, A.A.S. Violência urbana: um problema social. Revista de Sociologia da USP. São Paulo, V. 10, n. 1, p. 105-119. Maio, 1998.

LANG, J.A. et al. Adult psychopathology and intimate partner violence among survivors of childhood maltreatment. Journal of Interpessoal Violence, v. 19, n. 10, p. 1102-18, 2004.

LASCH, C. Refúgio num mundo sem coração: a família: santuário ou instituição sitiada. São Paulo: Paz e Terra, 1991. 252 p.

LIZUKA, T. et al. Midfacial Fractures in Pediatric Patients. Arch Otolaryngol Head Neck Surg. v. 121, n.12, p.1366-71, december, 1995.

MACEDO, J.L.S. et al. Perfil epidemiológico do trauma de face dos pacientes atendidos no pronto socorro de um hospital público. Revista do Colégio Brasileiro de Cirurgia.v.35, n.1, jan/ fev, 2008.

MANGANELLO- SOUZA, L.C. Trauma de Partes Moles: Princípios de Tratamento dos Ferimentos Cutâneos. In: MANGANELLO-SOUZA, L.C.; LUZ, J.G.C. Tratamento Cirúrgico do Trauma Bucomaxilofacial. $3^{\text {a }}$ ed, São Paulo, Roca, 2006, 340p.

MARGOLIN, G.; GORDIS, E.B. The Effects of Family and Community Violence on Children. Annual Review of Psychology, v. 51, p. 445-479, feb. 2000.

MENEZES, M.M. et al. Prevalência de traumatismos maxilofaciais e dentais em pacientes atendidos no pronto socorro municipal de São José dos Campos/SP. Revista Odonto Ciência, Porto Alegre, v. 22, n. 57, julho/ setembro, 2007.

MODOLIN, M.L.A.; MANGANELLO-SOUZA, L.C.; MAUAD JUNIOR, R.J. Cicatrização das Feridas. In: MANGANELLO-SOUZA, L.C.; LUZ, J.G.C. (Org.). Tratamento Cirúrgico do Trauma Bucomaxilofacial. $3^{\text {a }}$ ed, São Paulo, Roca, 2006, 340p.

MONTOVANI, J.C. et al. Etiologia e incidência das fraturas faciais em adultos e crianças: experiência em 513 casos. Revista brasileira de otorrinolaringologia. v.72, n.2, p. 235-241, 2006. 
NAGIN, D.; TREMBLAY, R.E. Trajectories of boys'Physical Agrgression, opposition, and Hyperatctivity on the Path to Physically Violent and Nonviolent Juvenile Delinquency. Child Development, v. 70, n. 5, p. 1181-1196, jan. 2003.

ORGANIZAÇÃO MUDIAL DA SAÚDE. Classificação estatística internacional de doenças e problemas relacionados à saúde. 10a rev. São Paulo: Centro Colaborador da Organização Mundial de Saúde para a Classificação de Doenças em Português, 1995.

ORGANIZAÇÃO DAS NAÇÕES UNIDAS. Declaração Universal dos Direitos Humanos, 1948. Disponível em: http://www.onu-brasil.org.br/documentos_direitoshumanos.php.

Declaração Universal dos Direitos da Criança, 1959. Disponível em:http://www. onu-brasil.org.br/doc_crianca.php. Acesso em: set 2008.

PAULA, C.S. et al. Saúde mental e violência entre estudantes da sexta série de um município paulista. Revista de Saúde Pública, v. 42, n.3, p.524-528, 2008.

PAVIANI, A.; FERREIRA, I.C.B.; BARRETO, F.F.P. (Org.). Brasília: dimensões da violência urbana. Brasília, Editora Universidade de Brasília, 2005, 378.

PINHEIRO, P.S.; ALMEIDA, G.A. Violência Urbana, São Paulo: Publifolha, 2003, p. 87. PORTOLAN, M.; TORRIANI, M.A. Estudo de prevalência das fraturas bucomaxilofaciais na região de Pelotas. Revista Odonto Ciência. Porto Alegre, v. 20, n.47, jan/ mar, 2005.

PREFEITURA DE BELO HORIZONTE. Área total, população e densidade demográfica, Brasil, MG, RMBH, BH - 2007. Disponível em http://portalpbh.pbh.gov.br/pbh/ecp/ comunidade.do? evento = portlet \&pIdPlc=ecpTaxonomiaMenuPortal\&app = estatisticas \&ta $\mathrm{x}=9086 \&$ lang=pt_BR\&pg=5922\&taxp=0\&. Acesso em: 10 jan 2009.

PRIGOZEN, J.M. et al. All-terrain Vehicle-Related Maxillofacial Trauma in the Pediatric Population. Journal of Oral and Maxillofacial Surgery, v. 64, n. 9, p.1333-1337, September 2006. RAHMAN, R.A. Et al. Maxillofacial trauma pediatric patients in Malaysia: A retrospective study from 1999 to 2001 in three hospitals. International Journal of Pediatric Otorhinolaryngology, v. 71, n. 6, p. 929-936, june 2007.

RIBAS, G.C. Traumatismo Cranioencefálico. In MANGANELLO-SOUZA, L.C.; LUZ, J.G.C. (Org.). Tratamento Cirúrgico do Trauma Bucomaxilofacial. $3^{\text {a }}$ ed, São Paulo, Roca, 2006, 340p.

RIBEIRO, J. C. A.; CHAVEIRO, E.F. Violência urbana, espaço urbano e subjetividade: uma leitura geográfica da violência urbana cotidiana. 3th ed. Revista Mirante, v. 1, n. 2, p. $1-17,2007$.

RIBEIRO, M.F.P; GÓES, P.S.A. Trauma maxilofacial. In: ANTUNES, J.L.F.; PERES, M.A.; CRIVELLO JÚNIOR, O. (Ed.). Fundamentos de Odontologia. Epidemiologia da Saúde bucal. Rio de Janeiro: Guanabara Koogan, 2006, 441p. 
RIDLEY, M. As origens da virtude: um estudo biológico da solidariedade. Rio de Janeiro: Record, 2000.

RODRIGUES, E.A.P. A Dimensão social da violência infanto-juvenil. Serviço Social em Revista, Londrina: UEL, v. 2, n. 1, p. 77-104, Jul. /Dez. 1999.

SALZINGER, S. et al. Aggressive behavior in response to violence exposure: is it adaptative for middle-school children? Journal of Community Psycology, v. 36, n. 8, p. 1008-1025, oct., 2008. SARMENTO, D.J.S.; CAVALCANTI, A.L.; SANTOS, J.A. Características e distribuição das fraturas mandibulares por causas externas: estudo retrospectivo. Pesquisa Brasileira de Odontopediatria e Clínica Integrada, João Pessoa, v. 7, n. 2, p. 139-144, maio/ agosto, 2007. STRON, C. Injuries due to violent crimes. Med Sci Law, London, v. 32, n. 2, p. 123-132, Apr. 1992.

VILHENA, J.; MAIA, M.V.C.M.. Agressividade e violência: reflexões acerca do comportamento anti-social e sua inscrição na cultura contemporânea. Revista Mal-Estar e Sociedade, Fortaleza, v. 2, n.2, p. 27-58, set. 2002.

ZACHARIADES, N.; PAPAVASSILIOU, D.; KOUMOURA, F. Fractures of the facial skeleton in children. Journal of Craniomaxillofacial Surgery, v. 18, p. 151, 1990.

WINNICOTT, D. W. Natureza e origens da tendência anti-social. In: . Privação e delinquência. São Paulo: Martins Fontes, 1987.

\section{Nota}

${ }^{1}$ Este artigo é resultado do trabalho de dissertação de mestrado defendida na Faculdade de Odontologia da UFMG. 


\section{The urban violence against children and adolescents in Belo Horizonte: a story told through the maxillofacial traumas}

The maxillofacial traumas resulting from violence against children and adolescents have physical and psychical impact on their lives, because of the deformities and the injuries on their faces. This study aims to identify the prevalence of maxillofacial traumas in children and adolescents caused by urban violence in Belo Horizonte, Brazil. The study was conducted at Hospital Municipal Odilon Behrens, the primary reference hospital in this type of care in Belo Horizonte. We collected records of victims attended between January and December 2007. Among children and adolescents, the most recurring violence event was physical aggression, 44,2\% and 64.7\% respectively. Among children, the most common type was the dental-alveolar trauma $(53.8 \%)$, while among adolescents, soft tissues trauma (47.5\%). The highest number of occurrences was at night, for both children $(84.6 \%)$ and adolescents $(74.8 \%)$. The most victimized gender was masculine, children (63.5\%), and adolescents (68.3\%). Appropriate strategies to indentify violent events, as well as the aggressor himself, are necessary for planning better protection mechanisms for children and adolescents, since the violence suffered by this group in Brazil, considering the complexity of this stage of life, takes a gloomy picture, deconstructing their development, sociability, and undermining the victims' perspective about themselves and the world around them.

> Key words: maxillofacial traumas, violence, children, adolescents. 